\title{
Fortuitous Case of Undifferentiated High Grade Uterine Sarcoma- A Rare Case Report from Pakistan
}

\author{
Ayesha Shahid ${ }^{1 *}$, Amna Javed ${ }^{2}$, Muhammad Ikram³, Makiya Sharif' and Rukiya Tariq ${ }^{4}$ \\ 'Trainee Resident, Department of Obstetrics and Gynecology, Shaikh Zayed Hospital, \\ Lahore-54000, Pakistan; ayeshashahidred@gmail.com \\ ${ }^{2}$ Assistant Professor, Department of Obstetrics and Gynecology, Shaikh Zayed Hospital, Lahore - 54000, Pakistan \\ ${ }^{3}$ Head of Department, Department of Obstetrics and Gynecology, Shaikh Zayed Hospital, Lahore - 54000, Pakistan \\ ${ }^{4} \mathrm{M}$. Phil., Public Health, Department of Public Health, University of the Punjab, Lahore -54000 , Pakistan
}

\begin{abstract}
Pre-operative diagnosis of fortuitous rare tumors require careful clinico-pathological review; representing challenges to diagnose sarcomas in clinical practice especially in developing countries. Studies are needed that signifies their clear representation.
\end{abstract}

Keywords: Chemotherapy, Female Genital Tract Malignancies, Recurrence, Uterine Sarcoma

\section{Introduction}

Uterine sarcomas are rare tumors derived from mesenchymal tumors ${ }^{1,2}$, predominantly affecting women of reproductive age with symptoms; uterine bleeding, pelvic pain and/or pelvic mass ${ }^{3}$ associated with poor prognosis and remain undiagnosed preoperatively ${ }^{4,5}$. Thus, attributed to $1 \%$ of female genital tract malignancies and $3 \%$ to $7 \%$ of uterine cancers in them ${ }^{6,7}$. The existing case reports unexpected finding of undifferentiated uterine sarcoma. Verbal as well as written informed consent for this study was obtained from the patient.

\section{Case Report}

A 30 years old woman para 1, with four months history of heavy menstrual bleeding and lower abdominal pain presented in Outpatient Department (OPD) of Obstetrics and Gynecology, Shaikh Zayed Hospital, Lahore. Abdo minal examination showed 22 weeks size mass, tender, firm in consistency with restricted mobility occupying lower abdomen and lower limit was not accessible. While, on speculum examination, vagina was filled with mass, firm to hard in consistency with necrotic areas, cervix was not visible due to the presence of substantial mass. Precise origin of mass was not definite but seemed to be originated from cervix/lower segment of uterus. Provisional diagnosis of pedunculated leiomyoma was made.

Ultrasonographic examination revealed multiple fibroids distorting uterine cavity with two large fibroids one in anterior upper wall of uterus with cystic changes measuring $6.7 \times 6.1 \mathrm{~cm}$ and other in posterior wall in lower region measuring $7 \times 6.2 \mathrm{~cm}$. No adnexal pathology was observed. With clinico-radiological findings, cumulative approach for myomectomy was premeditated. For this reason, surgery was initially started vaginally, but intraoperatively massive mass in vagina seems to be adherent to lower segment of uterus and cervix making it inseparable thus, abdominal approach proceeded. During laparotomy, normal size uterus with almost $18 \times 10 \mathrm{~cm}$ necrotic fibroid completely occupying lower uterine segment pushing uterus upward was noted. Therefore, hysterectomy with conservation of ovaries was performed with removal of all the noticeable disease portion.

Histopathological examination on cut section of uterus showed two greyish white necrotic endometrial and cervical growths involving full thickness of myometrium extending up to one sided parametrium and cervix and invading it measuring $6 \times 6 \times 5 \mathrm{~cm}$ each. Microscopic examination

*Author for correspondence 
revealed poorly differentiated high-grade uterine sarcoma as shown in Figure 1. Immunohistochemistry is positive for Cyclin-D1 focal positive $(<70 \%)$ and WT1-cytoplasmic staining and negative for Desmin, CD10, CD117, ER, CYTOKERATIN AE1/AE3, ASMA, h-Caldesmon.

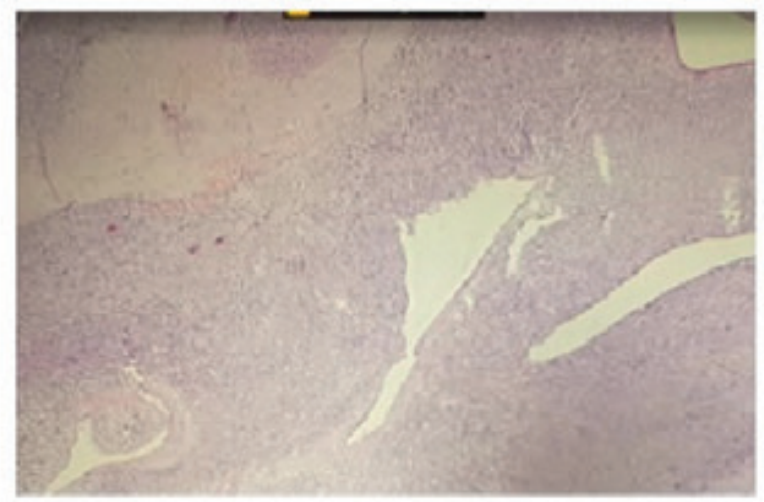

Figure 1. Low power field shows malignant spindle cell neoplasm with herring bone pattern and dilated vascular channels.

Patient was referred to Institute of Nuclear Medicine and Oncology Lahore (INMOL) Hospital, for auxiliary opinion and management concerning chemotherapy/radiotherapy with or without need of second surgery. Patient had lost to follow up due to socioeconomic issues. After 3 months of hysterectomy, patient started feeling lower abdominal pain and abdominal distention and presented once again in Shaikh Zayed Hospital for follow up, had urgent CT scan of abdomen, pelvis and lungs showing pelvic mass extending upward up to left lumber region with enlarged liver, abdominal ascites and metastasis in lungs.

Patient consulted INMOL Hospital for opinion and they proceeded with chemotherapy causing abrupt deterioration in renal functions of patient and were referred to Shaikh Zayed Hospital for supportive management. This unfortunate, young patient did not cope with life and expired during treatment in only eight months of disease span.

\section{Discussion}

The dilemma of leiomyosarcoma is that review of literature suggests its rarity with aggressive nature and high risk of recurrence and death regardless of its stage of presentation with no absolute preoperative diagnostic confirmation ${ }^{4,5}$. Imaging modalities that are commonly used including ultrasound and MRI cannot accurately and precisely differentiate between leiomyoma and leiomyosarcoma although progress has been made ${ }^{1}$. In our case, malignancy was diagnosed on histopathology specimen after surgery followed by recurrence with metastasis and poor prognosis due to lost to follow up. Thus, these study findings were found significant and congruent with research conducted by Nankali and his co-associates ${ }^{4}$.

\section{Conclusion}

The study concluded that the rarity of leiomyosarcoma and its similarities to benign tumor on ultrasonographic imaging, its aggressive nature with poor prognosis, high rate of recurrence and diagnostic challenge when analyzing patients pre-operatively is still a dilemma. Hence, suspicious looking fibroid must be evaluated accordingly.

\section{Ethical Approval}

Ethical approval was taken from Gynecology and Obstetrics Department, Hospital ethical committee.

\section{Acknowledgement}

We authors thank Mr. Obaid Ullah Ahmad for his massive effort in digital assistance making our case proficient.

\section{Conflicts of Interest}

The authors have no conflicts of interest.

\section{Funding Disclosure}

None.

\section{Data Availability Statement}

None.

\section{References}

1. Benson C, Miah AB. Uterine sarcoma-current pers pectives. International Journal of Women's Health. 2017; 9:597-606.PMid:28919822 PMCid:PMC5587218. https://www.ncbi.nlm.nih.gov/pmc/articles/PMC 5587218/ https://doi.org/10.2147/IJWH.S117754

2. Gremel G, Liew M, Hamzei F, Hardell E, Selling J, Ghaderi M, Stemme S, Ponten F, Carlson JW. A prognosis based classification of undifferentiated uter ine sarcomas: Identification of mitotic index, hormone receptors and YWHAE-FAM22 translocation status as predictors of survival. International Journal of Cancer. 2015; 136(7):1608-18. PMid:25130488. https://online library.wiley.com/doi/full/10.1002/ijc.29141 https://do i.org/10.1002/ijc.29141

3. KhanAT,ShehmarM,GuptaJK.Uterinefibroids:Current perspectives. International Journal of Women's Health. 2014; 6:95-114. PMid:24511243 PMCid:PMC3914832. 
https://www.ncbi.nlm.nih.gov/pmc/articles/P MC3914832/ https://doi.org/10.2147/IJWH.S51083

4. Nankali A, Keshavarzi F,Ataee M. A rare case of uterine leiomyosarcoma in a virgin woman: A case report. Journal of Biology and Today's World. 2014; 3(2):49-52. https://www.iomcworld.org/abstract/a-rare-case-ofuterine-leiomyosarcoma-in-a-virgin-woman-a-casereport-3332.html https://doi.org/10.15412/J.JBTW.010 30205

5. Odejinmi F, Agarwal N, Maclaran K,Oliver R. Should we abandon all conservative treatments for uterine fibroids? The problem with leiomyosarcomas. Women's
Health. 2015; 11(2):151-9. PMid:25776289. https:// journals.sagepub.com/doi/full/10.2217/WHE.14.71 https://doi.org/10.2217/WHE.14.71

6. Chandel S, Nigam A. Spindle cell sarcoma of uterine cervix-A rare case report: International journal of scientific and research publications: 2014; 4(5): 1-3. <http://www. ijsrp.org/research-paper-0514/ijsrp-p29118.pdf

7. Mbatani N, Olawaiye AB, Prat J. Uterine sarcomas. InternationalJournalofGynecologyandObstetrics.2018; 143:51-8. PMid:30306577. https://obgyn.onlinelibrary. wiley.com/doi/full/10.1002/ijgo.12613 https:/doi.org/1 0.1002/ijgo. 12613 